\title{
Gravitational displacement of submacular haemorrhage in patients with age-related macular disease
}

\author{
Gurkan Erdogan $^{1} \cdot$ Asli Kirmaci $\mathbb{1}^{1} \cdot$ Irfan Perente $^{1} \cdot$ Ozgur Artunay $^{1}$ \\ Received: 24 February 2019 / Revised: 10 October 2019 / Accepted: 18 October 2019 / Published online: 2 December 2019 \\ (c) The Author(s), under exclusive licence to The Royal College of Ophthalmologists 2019
}

\begin{abstract}
Objectives To investigate the efficacy of gravitational displacement following vitrectomy for the patients with submacular haemorrhage (SMH) secondary to age-related macular degeneration (AMD).

Methods Retrospective, interventional series of nine consecutive AMD patients with SMH. All patients underwent pars plana vitrectomy (PPV) combined with subretinal tissue plasminogen activator (t-PA) and intravitreal anti-vascular endothelial growth factor (anti-VEGF) injection without any tamponade. The patients were positioned according to the location and distribution of SMH postoperatively in order to provide the maximum gravitational force effect to displace. Outcome measures were the change in visual acuity, the displacement in SMH, and the occurrence of per- and postoperative complications.

Results Complete displacement of haemorrhage occurred in all patients postoperatively. The mean preoperative and postoperative best-corrected visual acuity (BCVA) at the last visit were 2.46 and $1.7 \log \mathrm{MAR}$, respectively, after a mean followup of 10.4 months (Range: 3-18 months) ( $p=0.045$ ). The mean duration of haemorrhage was 15.3 days (range: 3-40 days). The recurrence of SMH was observed in two (22\%) patients and there were no other postoperative complications.

Conclusion Gravitational displacement following PPV combined with subretinal t-PA and intravitreal anti-VEGF injection can be considered as an effective surgical intervention in selected AMD patients with SMH.
\end{abstract}

\section{Introduction}

Submacular haemorrhage (SMH) is a devastating complication of neovascular age-related macular degeneration (AMD) that causes irreversible vision loss by destroying the photoreceptors if left untreated [1]. Damage to the photoreceptors begins early and is directly proportional to the duration of exposure $[2,3]$. The technique applied becomes more successful, if haemorrhage is rapidly displaced from central submacular area. Although there are several techniques available, no consensus exists. Submacular injection of tissue plasminogen activator (t-PA) with pars plana

Supplementary information The online version of this article (https:// doi.org/10.1038/s41433-019-0720-8) contains supplementary material, which is available to authorised users.

Gurkan Erdogan

erdogangurkan@yahoo.com

1 University of Health Sciences Beyoglu Eye Training and Research Hospital, Istanbul, Turkey vitrectomy (PPV) might be considered as an effective method. Postoperative positioning, types and amounts of intravitreal gas tamponades are varied among the different approaches in this method; yet the common feature is the formation of a macular detachment as an expected result of subretinal injection [4-8].

The role of gravitational force in the displacement of SMH was emphasised in the literature previously $[7,8]$. We considered the space under the macular detachment as an important factor that led to the displacement of $\mathrm{SMH}$, which allows the movement of the liquefied haemorrhage with the aid of gravitational forces rather than the pressure effect of the applied tamponade. We performed PPV with submacular t-PA injection without intraocular tamponade and instructed patients to either sit in an upright position or to lie on the temporal side of the affected eye, according to the location and distribution of the haemorrhage. Following this SMHs were successfully displaced in all of the cases. According to our approach, neither prone positioning nor intraocular tamponade is necessary, and the treatment results can be obtained in the early postoperative period. 


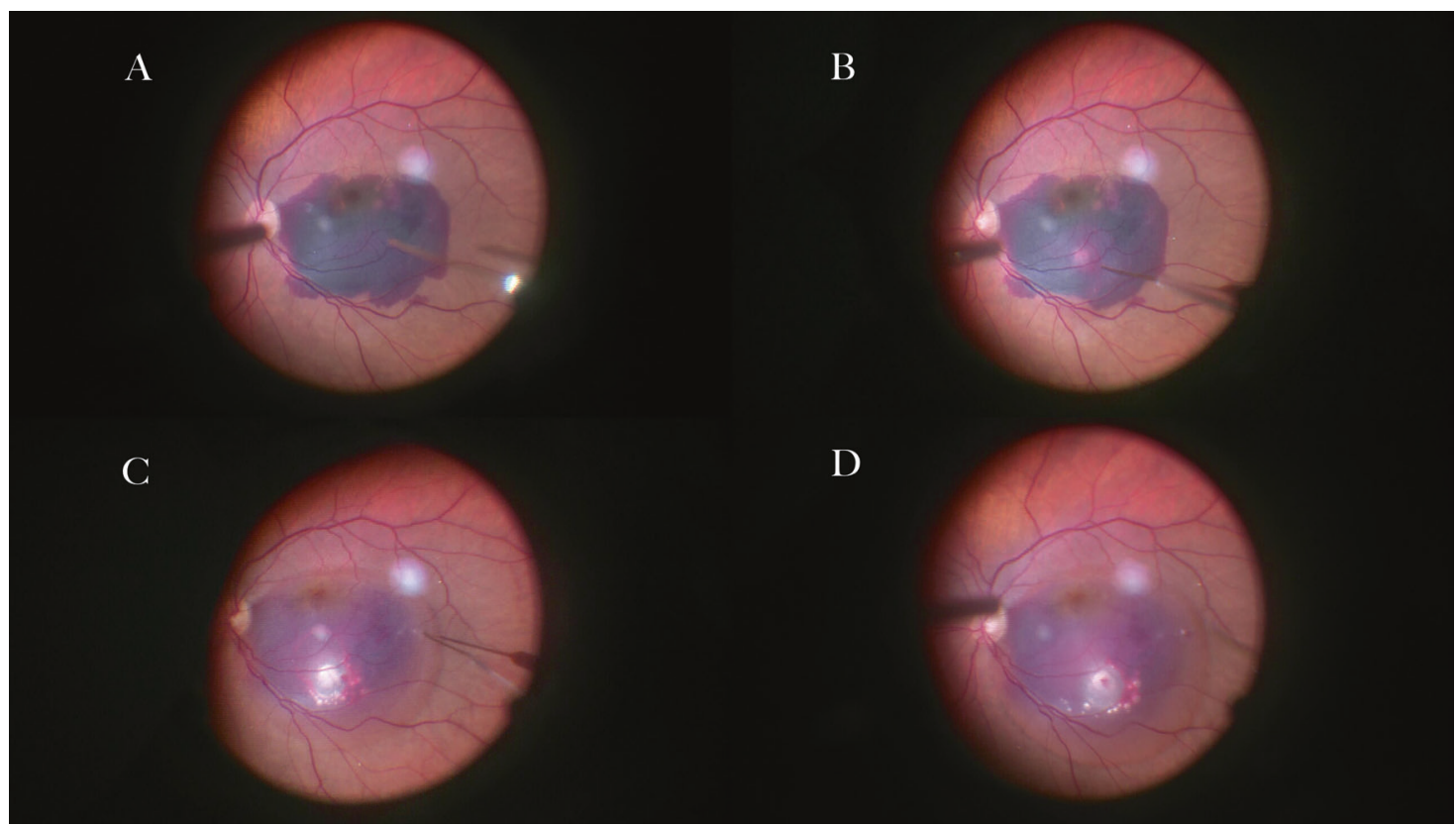

Fig. 1 Stages of the macular detachment creation. a Configuration of haemorrhage. b First subretinal injection of t-PA. c Second subretinal injection of t-PA. d Macular detachment

\section{Materials and methods}

This study is a retrospective interventional consecutive case series of patients with SMH secondary to neovascular AMD. The patients included in this study, underwent PPV with subretinal t-PA injection and intravitreal anti-VEGF injection between September 2016 and October 2017. The study protocol was approved by the local ethics committee and adhered to the tenets of the Declaration of Helsinki. Informed consents were obtained from all of the patients. Demographic features, duration and aetiology of SMH, and preoperative treatment with anti-VEGF agents, if exists, were recorded for each patient. At each visit, the patients underwent ophthalmologic examination including bestcorrected visual acuity (BCVA) evaluation using the Snellen chart, intraocular pressure measurement with Goldmann applanation tonometry, and fundus examination with indirect ophthalmoscopy. Besides, colour fundus photographs and optical coherence tomography (OCT) images were taken for each case. Fundus fluorescein angiography (Heidelberg Spectralis HRA + OCT; Heidelberg Engineering, Heidelberg, Germany) was performed at the discretion of the physician.

For each patient, the location and distribution of the subretinal blood in the posterior pole was described preoperatively, the appropriate direction to displace the subretinal blood away from the central macula was determined and the surgery was planned accordingly. Massive SMHs extending beyond superior arcade and optic disc were excluded. Following a 23 gauge PPV (Alcon Constellation ;
Alcon Laboratories, Fort Worth, TX, USA), a posterior vitreous detachment was performed, if not already present. Approximately $0.1-0.4 \mathrm{ml}$ of t-PA was injected into the subretinal space by 41 gauge flexible cannula at doses of $25 \mu \mathrm{g} / 0.1 \mathrm{~mL}$, not exceeding $100 \mu \mathrm{g}$ in total. Two or more injections were performed through separate locations and a macular detachment with a sufficient subretinal space was created to allow haemorrhage to displace. We expanded the detachment even more towards the direction in which we planned the haemorrhage to shift and exceeding the borders of haemorrhage 1-2 OD diameter distance. (Fig. 1) (Video). No intraocular tamponade was used at the end of the surgery. The operation was completed after the intraocular anti-VEGF injection through the pars plana with a $30-\mathrm{G}$ needle. The patients were advised to take supine position postoperatively in order t-PA to liquefy the haemorrhage, for $1 \mathrm{~h}$; after which they were positioned upright or lying down on the temporal side of the affected eye, for 1-3 days. The postoperative positions of the patients were determined according to the direction of displacement planned preoperatively. Intraoperative and postoperative complications were evaluated, and postoperative follow-up examinations of the patients on day $1,3,7,14$, month 1 and also on following visits were recorded. More frequent visits were performed for the cases in which the haemorrhage was successfully displaced, but the subretinal fluid persisted, and the resolution time of subretinal fluid for each case was recorded. Any patient showing signs of activation in the postoperative period received additional treatment accordingly. 


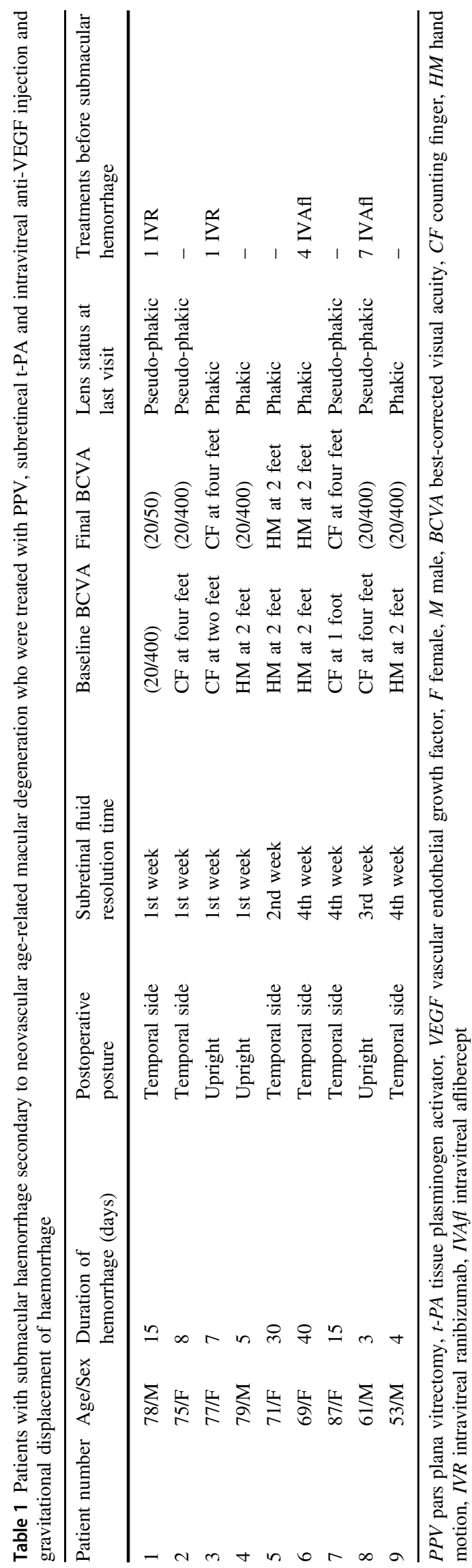

\section{Results}

Of the nine eyes from nine patients who met our inclusion criteria, five $(55.6 \%)$ were female and four $(44.4 \%)$ were male with a mean age of 72.2 years (range: 53-87 years). Four of the patients received intravitreal anti-VEGF therapy before the appearance of SMH. All of the cases included in our study underwent PPV, subretinal t-PA and intravitreal anti-VEGF injection and gravitational displacement of haemorrhage for SMH secondary to AMD. The mean duration of haemorrhage from the starting of symptoms to the time of the surgery was 15.3 days (range: 3-40 days). Demographics, patient characteristics, baseline, and final examination findings are summarised in Table 1.

The preoperative BCVA ranged from hand motion (HM) to 20/400 (mean 20/2208-2.46 logMAR), and the postoperative BCVA at the last follow-up ranged from HM to 20/50 (mean 20/289-1.7 logMAR). The increase in BCVA after the surgery was found statistically significant $(p=$ 0.045 , Paired sample $t$ test). There was a strong positive correlation between the initial BCVA and the final BCVA ( $p=0.045, r=0.678$, Pearson test). After the successful displacement of haemorrhages, the increase in BCVA was significant in five (55.6\%) of the cases (Patient number 1, 2, $4,8,9)$, and limited in two $(22.2 \%)$ of the cases (Patient number 3,7); whereas the BCVA remained stable in two $(22.2 \%)$ of the cases (Patient number 5, 6) (Table 1). The two patients with the stable BCVA were also the patients with the longest duration of haemorrhage. There was a strong negative correlation between the duration of haemorrhage and the final BCVA $(p=0.035, r=-0.703$, Pearson test). At the time of admission to our clinic, six patients were phakic and three were pseudo-phakic. First patient underwent cataract surgery during the follow-up period. (Table 1) The sixth and eighth patients were followed up in our clinic preoperatively and the other patients were referred patients. The previous treatments before the SMH are presented in Table 1.

According to the location and distribution of SMH; three (33\%) of the patients were instructed to sit in upright position and six (67\%) of the patients to lie on the temporal side of the affected eye, postoperatively. In all of the cases, haemorrhages were displaced almost completely on the first day after the surgery. The minimal residual liquefied haemorrhage observed in some patients resolved gradually by both being displaced inferiorly and being absorbed, through the first 2-3 weeks postoperatively (Figs. 2 and 3). After the complete displacement of SMH, the residual subretinal fluid resolved in the first week after the surgery in four (44\%) of the patients; whereas it took between 2 and 4 weeks in five $(56 \%)$ of the patients (Patient number 5, 6, 7, 8, 9) (Fig. 4).

The mean follow-up time was 10.4 months (Range: 3-18 months). The recurrence of SMH was observed in two 


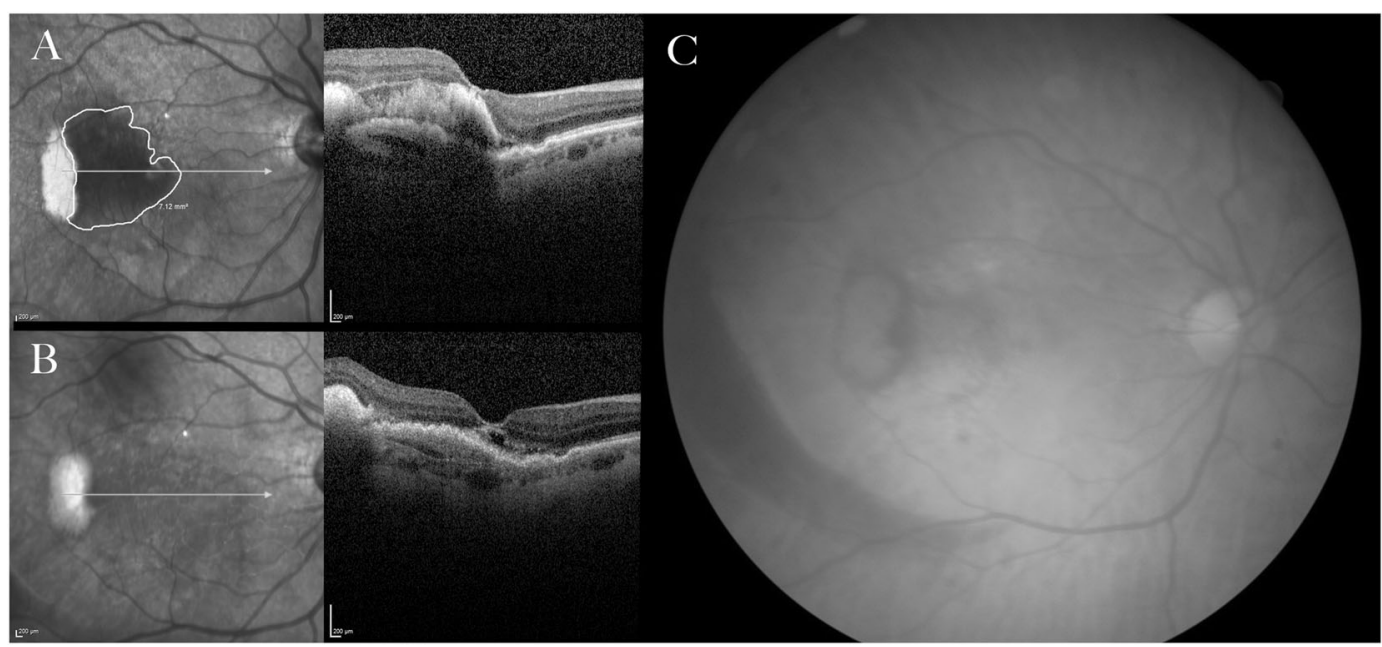

Fig. 2 Preoperative and postoperative findings of patient number 1. a Preoperative OCT. b Postoperative OCT (day 4). c Postoperative colour fundus photography (day 4)

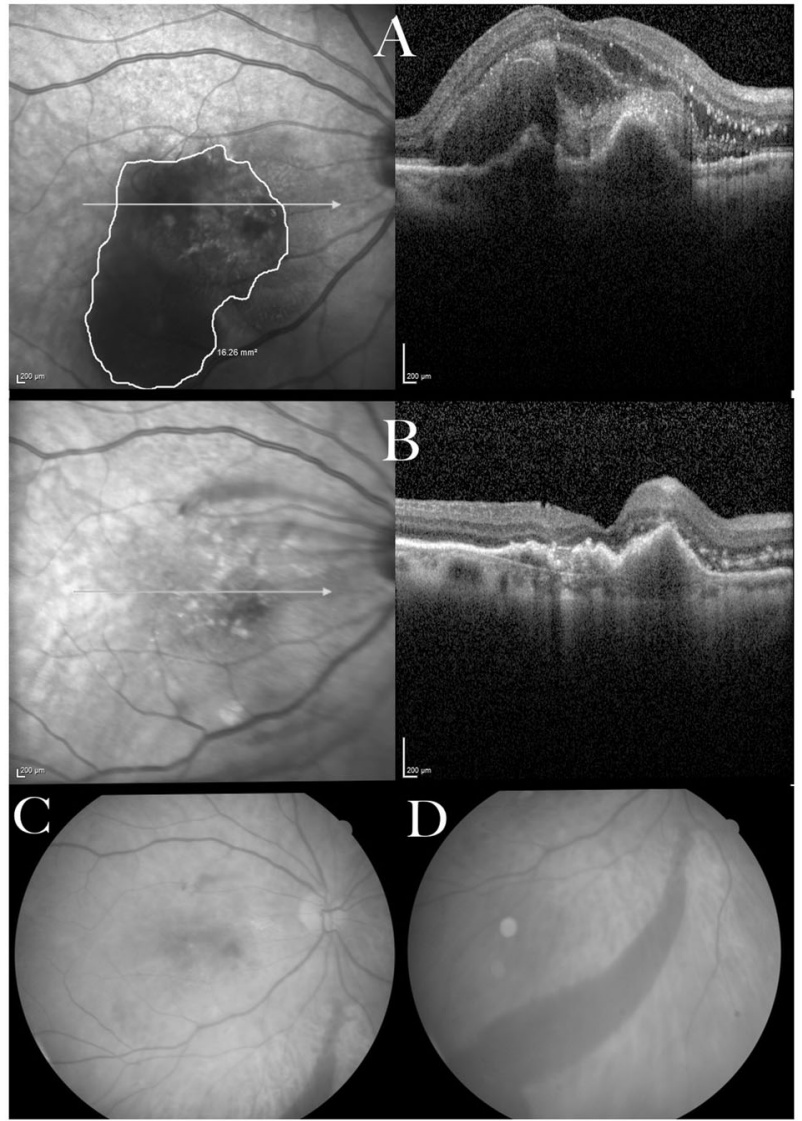

Fig. 3 Preoperative and postoperative findings of patient number 3. a Preoperative infrared image and OCT. b Postoperative infrared image and OCT (day 5). c) Postoperative colour fundus photography (day 5). d Postoperative colour fundus photography, inferior (day 5)

(22\%) of the patients on the postoperative follow-up visits at month 3 , and the same surgical procedure was repeated for these two patients. No additional postoperative complications were observed in any of the patients, including endophthalmitis or retinal detachment.

\section{Discussion}

The introduction of t-PA in the 1990s has provided a major step in the treatment of SMH [9, 10] and many different methods have been applied over years since then. Dissolving the clotted haemorrhage, t-PA makes it more mobile and absorbable by the liquefaction process. Application of gas tamponades with postoperative prone positioning have been performed in several techniques for pneumatic displacement; with either subretinal or intravitreal t-PA injection, with or without PPV [3-5]. The common feature of these techniques is adjusting the macula as the uppermost point of the eye by means of the prone position, and therefore replacing the SMH away from the central macula to the periphery, with the help of the force exerted by the gas tamponade.

The success rate of the procedures including the gas tamponade application and postoperative prone positioning is thought to be mainly determined by the patient's compliance and co-operation. The difficulty in providing the exact alignment of the gas bubble under the central macula, and the physical challenges in achieving the prone position in elderly patients, who frequently suffer from orthopaedic problems, are some of the drawbacks of these procedures. Moreover, the intravitreal gas tamponade obscures the detailed examination of the macula in the early postoperative period, especially in phakic patients. This causes a delay in the detection of any treatment failures and in the initiation of alternative retreatment methods; which, in turn, causes more photoreceptor damage that would otherwise be prevented by early intervention. 
Fig. 4 Resolution of subretinal fluid after the displacement of haemorrhage in patient number 7. a Preoperative OCT.

b Postoperative OCT (day 6). c Postoperative OCT (day 14). d Postoperative OCT (day 26)
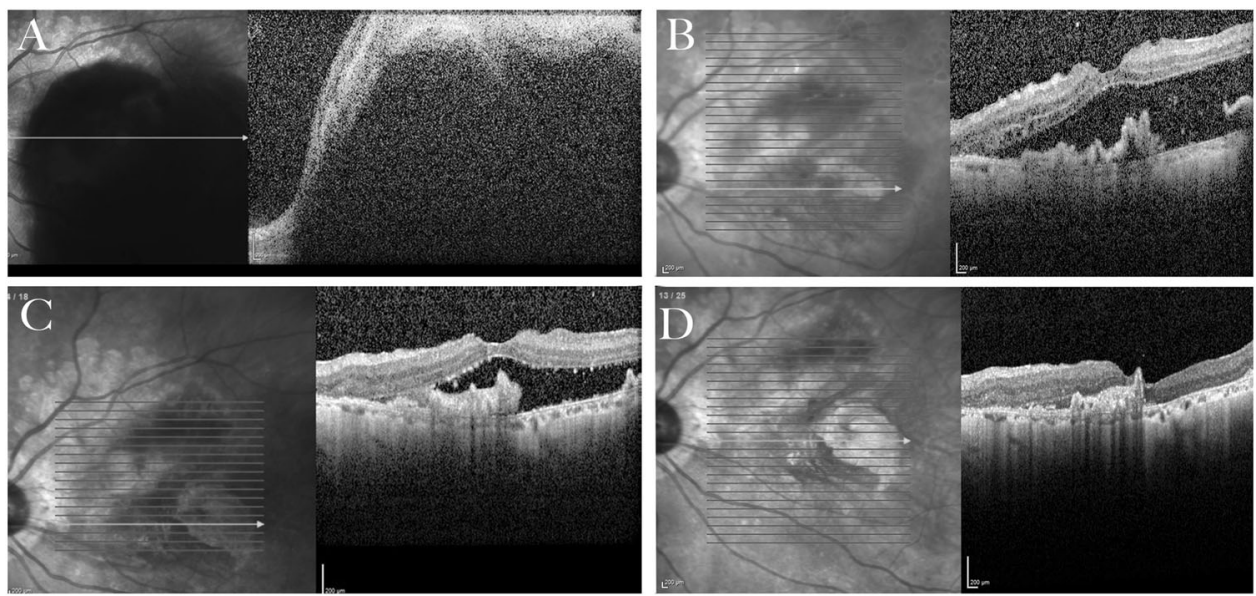

The direction of displacement of haemorrhage in patients, who underwent intravitreal gas tamponade procedure with t-PA administration and postoperative prone positioning, has been investigated in a small number of studies in the literature. In these studies, it has been shown that the displacement of haemorrhage was in the inferior direction in both of the approaches, in which the vitreous cavity was either completely or partially filled with gas [11-14]. The inferior shift of the haemorrhage in these approaches was different than the expected direction of shift provided by the gas tamponade, which is to the all directions to the periphery and away from the centre. Stopa et al. analysed the forces affecting the displacement of SMH and pointed out that surface tension and intraocular pressure produced by gas are uniformly distributed through the whole retinal surface. Since the effect of gas tamponade is equally distributed on the retina either with or without subretinal haemorrhage, it does not contribute to the displacement of haemorrhage in the eye full of gas. They stated that also in partially gas-filled eyes, the gas bubble is unlikely to overlap with SMH and unlikely to cause a pushing or rolling effect. They determined that the gravitational force is the most important factor responsible for the displacement of haemorrhage and advised avoiding the prone position in the postoperative period. They noted that the low buoyancy force of gas tamponade contributes to the gravitational forces and the haemorrhage displaces downwards more easily by its own weight in the gas-filled vitreous cavity [7]. Lincoff et al. reported that the gravitational force is most effective at the $40^{\circ}$ gaze down position in patients treated with expansile gas and relatively small bubbles, and observed that the displacement of haemorrhage takes place in a period of $\sim 1$ week [8]. In a previous study published from our clinic, SMHs were successfully replaced in the patient group who applied postoperative upright position after undergoing PPV with t-PA and gas application [6]. Sandhu et al. used air as a tamponade instead of expansile gas with subretinal t-PA injection and they did not give any posturing instruction during the daytime to the patients, they just asked them to lie on the side of the effected eye during sleep at night, in a similar way to our practice [15].

In the studies claiming the important effect of the gravitational forces on the SMH treatment, it was hypothesised that subretinal t-PA together with the liquefied haemorrhage provides a temporary retinal detachment in the adjacent retinal areas, through which the blood displaces. We agree that the main factor affecting the displacement of haemorrhage is the gravitational force; however, we consider that the space of macular detachment created by the subretinal tPA injection is crucial for the displacement of the haemorrhage. We realised that gravitational forces have two effects: One is to allow haemorrhage to settle to the lower part of the created macular detachment, and the other is to allow the detachment location to shift away from the macula. As the subretinal fluid is displaced, the detachment size decreases over time by being absorbed by the RPE. The location where the fluid is completely absorbed and the detachment disappears corresponds to the last point displacement has come and where the haemorrhagic particles remain trapped in the subretinal region.

In our technique, we created a macular detachment with a sufficient subretinal space and adequate width and aimed to move the haemorrhage away to a region distant from the central macula within the formed space of detachment. We observed that haemorrhagic suspension fluid in the subretinal region created by t-PA injection is more dense than the fluid in the vitreous cavity and allows the detachment to move to the periphery. Therefore, we avoided the application of gas in any of the cases.

We decided on the direction of shift by considering both the configuration of haemorrhage and the way to make the best use of gravitational forces. We applied postoperative positions as sitting upright in some patients and lying on the temporal side of the affected eye in the others; according to the haemorrhage location. We observed that the central 
SMH was displaced on the first day in all of the patients; however, it took $<1$ week in four cases, and between 2 and 4 weeks in five cases for the subretinal fluid to be completely resolved. The period of time for absorption of residual subretinal fluid was related to the amount of functional RPE. In the patients with scar tissue and widespread retinal atrophy, the removal of the subretinal fluid took longer. Also the visual acuity was lower in the cases with central macula affected by atrophy and scar tissue. Although RPE and retina was affected to a certain extent due to the natural course of AMD in all patients, haemorrhage also had a significant contribution to the postoperative visual outcome in proportion to the length of duration under the submacular region. Although we do not know about the exact status of all patients prior to $\mathrm{SMH}$, the patients with the lowest postoperative visual acuity were the fifth and sixth patients with the longest duration of haemorrhage in the submacular region. Furthermore, subretinal t-PA facilitates absorption of liquefied haemorrhage and absorption has a specific contribution on removal haemorrhage.

The advantages of our approach are that it has a short duration for the application, enables quick evaluation of the treatment response making any early intervention possible, requires easier postoperative positioning, carries advantages of not using tamponade such as early visual recovery and the absence of tamponade related complications. In conclusion; gravitational displacement following PPV combined with subretinal t-PA and intravitreal anti-VEGF injection can be considered as an effective and advantageous surgical intervention in selected AMD patients with SMH. Comparative studies with more cases are necessary to obtain real results of the procedure.

\section{Summary}

\section{What was known before}

- Gas tamponade is mandatory in order to displace the subretinal haemorrhage after subretinal t-PA application. Removal of submacular haemorrhage is provided with the help of displacing forces in preretinal space.

\section{What this study adds}

- Displacement of submacular haemorrhage can be achieved without the use of tamponades. Not only the preretinal space but also the subretinal space is important when displacing submacular haemorrhage. Gravitational forces are important in displacement of submacular haemorrhage.

\section{Compliance with ethical standards}

Conflict of interest The authors declare that they have no conflict of interest.

Publisher's note Springer Nature remains neutral with regard to jurisdictional claims in published maps and institutional affiliations.

\section{References}

1. Avery RL, Fekrat S, Hawkins BS, Bressler NM. Natural history of subfoveal subretinal hemorrhage in age-related macular degeneration. Retina. 1996;3:183-9.

2. Glatt H, Machemer R. Experimental subretinal hemorrhage in rabbits. Am J Ophthalmol. 1982;946:762-73.

3. González-López JJ, McGowan G, Chapman E, Yorston D. Vitrectomy with subretinal tissue plasminogen activator and ranibizumab for submacular haemorrhages secondary to agerelated macular degeneration: retrospective case series of 45 consecutive cases. Eye. 2016;30:929-35.

4. Gok M, Karabaş VL, Aslan MS, Kara O, Karaman S, Yenihayat F. Tissue plasminogen activator-assisted vitrectomy for submacular hemorrhage due to age-related macular degeneration. Indian J Ophthalmol. 2017;65:482-7.

5. Fassbender JM, Sherman MP, Barr CC, Schaal S. Tissue plasminogen activator for subfoveal hemorrhage due to age-related macular degeneretaion: comparison of three treatment modalities. Retina. 2016;3610:1860-5.

6. Kapran Z, Ozkaya A, Uyar OM. Hemorrhagic age-related macular degeneration managed with vitrectomy, subretinal injection of tissue plasminogen activator, gas tamponade, and upright positioning. Ophthalmic Surg Lasers Imaging Retin. 2013;44:471-6.

7. Stopa M, Lincoff A, Lincoff $\mathrm{H}$. Analysis of forces acting upon submacular hemorrhage in pneumatic displacement. Retina. 2007;27:370-4.

8. Lincoff H, Kreissig I, Stopa M, Uram D. A 40 degrees gaze down position for pneumatic displacement of submacular hemorrhage: clinical application and results. Retina. 2008;28:56-9.

9. Lewis H, Resnick SC, Flannery JG, Straatsma BR. Tissue plasminogen activator treatment of experimental subretinal hemorrhage. Am J Ophthalmol. 1991;111:197-204.

10. Lewis H. Intraoperative fibrinolysis of submacular hemorrhage with tissueplasminogen activator and surgical drainage. Am J Ophthalmol. 1994;118:559-68.

11. Heriot WJ. Intravitreal gas and tPA: an outpatient procedure for submacular hemorrhage. Chicago: AAO Annual Vitreoretinal Update; 1996.

12. Meyer CH, Scholl HP, Eter N, Helb HM, Holz FG. Combined treatment of acute subretinal haemorrhages with intravitreal recombined tissue plasminogen activator, expansile gas and bevacizumab: a retrospective pilot study. Acta Ophthalmol. 2008;86:490-4.

13. Abdelkader E, Yip KP, Cornish KS. Pneumatic displacement of submacular haemorrhage. Saudi J Ophthalmol. 2016;30:221-6.

14. Krepler K, Kruger A, Tittl M, Stur M, Wedrich A. Intravitreal injection of tissue plasminogen activator and gas in subretinal hemorrhage caused by age-related macular degeneration. Retina. 2000;20:251-6.

15. Sandhu SS, Manvikar S, Steel DH. Displacement of submacular hemorrhage associated with age-related macular degeneration using vitrectomy and submacular tPA injection followed by intravitreal ranibizumab. Clin Ophthalmol. 2010;21:637-42. 